\title{
Possibility of using local spline functions for estimating displacement values when observing deformations of buildings
}

\author{
Alexander Labuznov ${ }^{1, *}$ \\ ${ }^{1}$ Moscow State University of Civil Engineering, 26, Yaroslavskoye Shosse, 109377, Moscow, Russia
}

\begin{abstract}
This paper discusses the issue of determining the values of displacement points on the walls of buildings and structures during deformations using geodetic measurements of the values of displacements of adjacent points. It is proposed to use spline functions of one or two variables to calculate the offsets of points where no measurements were made, or where observations stopped starting from a certain stage. It is proposed to determine the spline coefficients either by interpolating the results from one observation cycle to arbitrary points, or by interpolating the observation results in previous cycles to points that were not observed in the new observation cycle. A specific example is used to evaluate the accuracy of the interpolation results and provide practical recommendations for using the methods in practical calculations. When considering the two-dimensional case, it is concluded that the approximation of the linear spline of two variables is not accurate enough, and it is proposed to use splines of a higher degree.
\end{abstract}

\section{Introduction}

When observing deformations of buildings, one of the problems is the need to observe a large number of points. In case of loss of the deformation mark, it becomes impossible to observe the same point in subsequent observation cycles. In this regard, it becomes important to consider questions about the possibility of determining the displacement of a point on the wall of a building from the results of observations of the remaining points for both the one-dimensional case and the plane. First of all, it is necessary to evaluate the accuracy of determining displacements by approximation methods (for example, when using the methods of spline functions). All measurements are assumed to be performed by geodetic methods.

To approximate the deformations of objects of significant area (for example, objects on the ground), quite satisfactory accuracy can be obtained by the finite element method with an appropriate choice of network shape and element sizes (see, for example, [1], [2]). The construction of surfaces, their approximation by splines based on the results of automatic measurement in various fields of engineering using modern software and computer equipment is a solved problem [3].

\footnotetext{
* Corresponding author: doptaganka@yandex.ru
} 


\section{Methods}

Let there be observations of displacements of deformation marks located on the surface of the building or structure, and let there be no singularities on the areas under consideration. Let us consider the surface being deformed as a graph of the function of two variables $y=f$ $(\mathrm{x}, \mathrm{h})$. From observations the values of function $\mathrm{y}$ are known at some regular (Consideration of only regular mesh is natural: as shown in [4], uniform grids are applicable for any types of surfaces, including features in the form of singularities) (deviations of distances between marks from the average value can be neglected) grid $\Delta$ specified by points selected during observations of deformations with coordinates $\left(\mathrm{x}_{\mathrm{i}}, \mathrm{h}_{\mathrm{i}}\right)$. In the case of observations of deformed surfaces, the function defining the surface is not known, nor are its derivatives known. The problem of approximation of an unknown function by its values at certain points can be solved in different ways, but from a practical point of view, the most convenient is approximation with the help of spline functions (There are many different algorithms for constructing splines; a fairly effective algorithm for constructing splines for complex surfaces is described, for example, in [5]). Building a spline for an unknown function $\mathrm{y}(\mathrm{x}, \mathrm{h})$ in the region of interest allows you to calculate its value at any point. This makes it possible to avoid excessive density in the location of observed points on the wall surface or, in case of loss of deformation mark, to make an estimate of displacements at the point where data from previous observation cycles are available.

When observing planned deformations (displacements), they are often limited by the location of deformation marks in one row; the arrangement of stamps in several tiers is used much less frequently. Accordingly, it is necessary to consider two fundamentally different cases - "flat", when the points lie on the same line, and "spatial" (deformation marks are located at significantly different heights). This article is devoted to the analysis of the first case.

Consider the "linear" case: placement of deformation marks is approximately the same line: marks lie on a curve close to the section of the wall surface by the oXY plane at time $t_{0}$ (Fig. 1). Then the planned shift of the mark in the direction perpendicular to the wall plane (along the oY axis) is a function of one variable $-\mathrm{x}$.

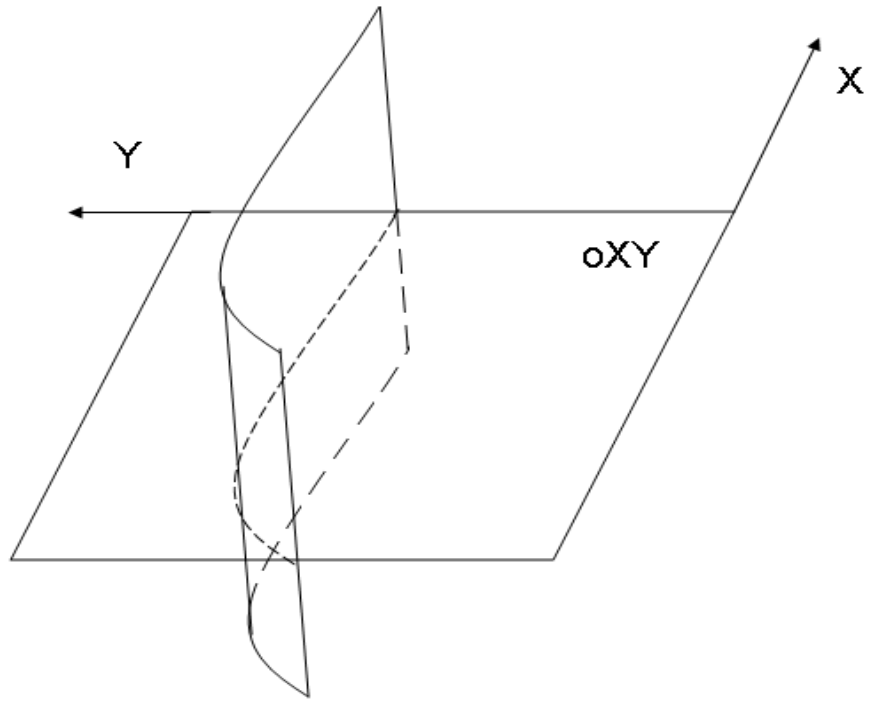

Fig. 1. Deformed wall surface. 
The standard approximation of an unknown function given in tabular form is by the Lagrange interpolation polynomial:

$$
L_{N}(x)=\frac{\sum_{i=1}^{N} f\left(x_{i}\right) \omega_{N}(x)}{\left[\left(x-x_{i}\right) \omega_{N}^{\prime}\left(x_{i}\right)\right]},
$$

wherein $\omega_{N}(x)=\prod_{i=1}^{N}\left(x-x_{i}\right)$.

However, when the degree of polynomial increases, the accumulation of errors due to rounding increases rapidly, so in practical cases it is more convenient to use splines functions composed of polynomials of the same degree defined each on its segment. The use of S-splines to significantly improve accuracy is difficult due to the requirement of fourth-order derivatives (i.e., $\mathrm{f}(\mathrm{x})$ belongs to class $\mathrm{C}^{4}[\mathrm{a}, \mathrm{b}]$, see, for example [6]).

The simplest interpolation suitable for use in strain observations will be a broken linear spline. In this case, the greatest error of such approach will have the top side fluctuation of function on a piece $\omega(\mathrm{f})=\max \left|\mathrm{f}\left(\mathrm{x}_{\mathrm{t}}\right)-\mathrm{f}\left(\mathrm{x}_{\mathrm{p}}\right)\right|, \mathrm{x}_{\mathrm{t}}, \mathrm{x}_{\mathrm{p}} \in\left[\mathrm{x}_{\mathrm{i}-1} ; \mathrm{x}_{\mathrm{i}}\right]$. It will be determined by the value $\max \partial=f_{i-1, i}\left(x_{i}\right)-\left(k_{i} x+b_{i}\right)$ on the set of segments $\left[x_{i-1} ; x_{i}\right], f_{i-1, i}\left(x_{i}\right)$ - interpolated function considered on this segment).

It is impossible to improve the estimate - the error of linear interpolation can be as close as possible to the value of $\omega(\mathrm{f})$ (Figure 2). In addition, the broken line does not reflect the basic geometric properties of the object: building walls, except for corners created according to the project, are usually smooth surfaces.

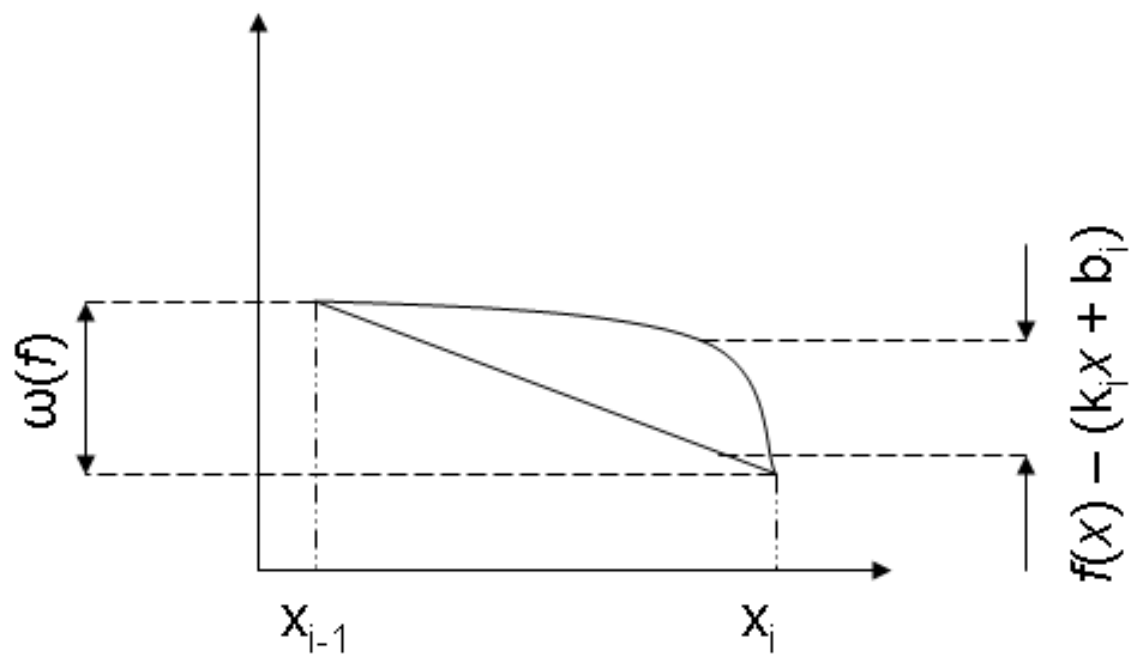

Fig. 2. Error of linear interpolation.

The most common in technical applications is the interpolation functions with a spline of the third degree ([7]). When interpolating with cubic Hermite splines, the interpolation error estimate is

$$
\Delta_{\mathrm{p}}=\frac{1}{16}\left(\max \left(\mathrm{x}_{\mathrm{i}}-\mathrm{x}_{\mathrm{i}-1}\right)\right)^{2}\left\|\mathrm{f}^{\prime \prime}(\mathrm{x})\right\|_{\infty}
$$

wherein $\left\|\mathrm{f}^{\prime \prime}(\mathrm{x})\right\|_{\infty}$ - essential supremum of the second derivative. 
Under the most general assumptions (existence of the first derivative, which is equivalent to the requirement of absence of cracks, seams in the wall, as well as angles within the line under consideration) the error does not exceed

$$
\Delta_{\mathrm{p}}=\frac{3 \max \left(\mathrm{x}_{\mathrm{i}}-\mathrm{x}_{\mathrm{i}-1}\right) \omega\left(\mathrm{f}^{\prime}\right)}{8^{4}} .
$$

When using cubic polynomials as approximating functions, there are two options. First: for each segment between the deformation marks on the wall of the building, a polynomial is selected; the second - one polynomial is constructed for several (two or three) adjacent segments.

However, when constructing a spline passing immediately through four points, it will not be possible, in the general case, to achieve the equality of the derivatives of polynomials at the boundaries of the segments. An exception may be the case when the surface at a point coinciding with the deformation mark has a kink and, accordingly, different (one-sided) derivatives. If this brings an increase in the accuracy of approximation in the middle of the segment, then the contradiction with the physical picture at the boundaries of the segment can be neglected - in these areas there is no practical need to calculate the displacements.

Let a cubic spline be built for each line segment. At the first and last split points

$$
\mathrm{y}^{\prime \prime}\left(\mathrm{x}_{0}\right)=\mathrm{y}^{\prime \prime}\left(\mathrm{x}_{\mathrm{n}}\right)=0
$$

For $\mathrm{n}+1$ points, we obtain $2 \mathrm{n}$ equations of the form

$$
\mathrm{f}_{\mathrm{i}-1, \mathrm{i}}\left(\mathrm{x}_{\mathrm{i}}\right)=\mathrm{y}_{\mathrm{i}},
$$

Then for $n-1$ equations of the form $\mathrm{f}_{\mathrm{i}-1, \mathrm{i}}\left(\mathrm{x}_{\mathrm{i}}\right)=\mathrm{f}_{\mathrm{i}, \mathrm{i}+1}\left(\mathrm{x}_{\mathrm{i}}\right)$, and two conditions on the second derivatives - total $3 n+1$ equations to determine $4 n$ coefficients $a_{i}, b_{i}, c_{i}, d_{i} f_{i-1, i}(x)=$ $a_{i} x^{3}+b_{i} x^{2}+c_{i} x+d_{i}$. Thus, $n-1$ more conditions (The requirement $S_{3}^{\prime}\left(x_{i}\right)=f_{i}$, which is part of the definition of Hermitian cubic splines, requires knowledge of the values of the derivatives $\mathrm{f}_{\mathrm{i}}$ at the points $\mathrm{x}_{\mathrm{i}}$; since the tangents to the wall surface at the points where the deformation marks are installed are not known, this requirement can be replaced by the requirement of equality of derivatives resulting from the absence of cracks and joints in the wall.) are needed (these splines are not Hermite). If you add the continuity requirement of the second derivative of $\mathrm{f}_{\mathrm{i}-1, \mathrm{i}}(\mathrm{x})$ functions, the task is reduced to the well-known task of constructing a function that takes known values at specified points and minimizes the functional $\int_{b}^{a}\left[f^{\prime \prime}(x)\right]^{2} d x$ - the task of potential energy of an elastic deformed rod with fixed points.

However, unlike the rod problem, the "potential energy" of a deformed wall is not necessarily minimal. This issue at present seems to have been studied quite insufficiently. Also, the second derivative can be discontinuous (Fig. 2), and then some additional ad hoc hypotheses are needed. In this case, the estimate of the accuracy of the approximation of actual displacement of the point, as already noted, is

$$
\Delta_{\mathrm{p}}=\frac{3 \max \left(\mathrm{x}_{\mathrm{i}}-\mathrm{x}_{\mathrm{i}-1}\right) \omega\left(\mathrm{f}^{\prime}\right)}{8} .
$$

Consider the second case: the interpolating polynomial of the third degree is built for two segments at once. The definition areas of two different functions can have only the ends of the lines in common (the number of lines must be even, respectively). Then each function $\mathrm{f}_{\mathrm{i}-1},{ }_{\mathrm{i}+1}(\mathrm{x})$ must satisfy three equations 


$$
\mathrm{f}_{\mathrm{i}-1, \mathrm{i}+1}\left(\mathrm{x}_{\mathrm{i}-1}\right)=\mathrm{y}_{\mathrm{i}-1}, \mathrm{f}_{\mathrm{i}-1, \mathrm{i}+1}\left(\mathrm{x}_{\mathrm{i}}\right)=\mathrm{y}_{\mathrm{i}}, \mathrm{f}_{\mathrm{i}-1, \mathrm{i}+1}\left(\mathrm{x}_{\mathrm{i}+1}\right)=\mathrm{y}_{\mathrm{i}+1}
$$

The number of deformation marks should be expressed by an odd number $2 \mathrm{k}+1$, then the number of equations of the form $\mathrm{f}_{\mathrm{i}-1}, \mathrm{i}+1\left(\mathrm{x}_{\mathrm{i}-1}\right)=\mathrm{y}_{\mathrm{i}-1}$, is $3 \mathrm{k}$, the requirement is $\mathrm{f}_{\mathrm{i}-2}, \mathrm{i}\left(\mathrm{x}_{\mathrm{i}}\right)=$ $\mathrm{f}_{\mathrm{i}, \mathrm{i}+2}\left(\mathrm{x}_{\mathrm{i}}\right)$ gives another $\mathrm{k}-1$ equation. Given that for $\mathrm{k}$ functions with 4 coefficients with powers of $\mathrm{x}, 4 \mathrm{k}$ equations are needed, until the system is definite, one equation is missing. The missing equation can be obtained from any additional condition, for example, the condition

$$
\sum a_{i}^{2}=\min
$$

Let a certain interpolating function be considered for a wall section with four deformation marks located on it. If we look for an interpolating function in the form of a cubic polynomial, without imposing any restrictions at the ends of the interval on either the function or its derivative, then the problem is well defined. If there are four points at which deformation observations were performed, the coefficients of the cubic spline can be determined

$$
f_{i-1, i}(x)=a_{i} x^{3}+b_{i} x^{2}+c_{i} x+d_{i}
$$

Let us evaluate the possibility of using a spline obtained on the basis of data from earlier observation cycles to "restore" the data at the point where observations in subsequent cycles were not performed. Let the coordinates of all deformation marks be determined in the $\mathrm{k}$-th observation cycle, and in the $\mathrm{k}+1$-th mark the number $\mathrm{m}$ is lost. Then you can use splines built on points from the cycle $\mathrm{k}$. The results of observations of four points in two randomly selected cycles (the interval between observations is more than one year) is given in table. 1 , lines 1,2 . The coordinate system used is local, common to all observation cycles. We calculate the coefficients of the cubic splines $f_{m-2, m-1}(x) f_{m}+1, m+2$ $(\mathrm{x})$, substitute the coordinates $\mathrm{x}_{\mathrm{i}}$ of the points from the cycle $\mathrm{k}+1$ from the intervals that do not contain the point $\mathrm{m}$ into the found functions.

Table 1. $\mathrm{X}$ and $\mathrm{Y}$ calculating.

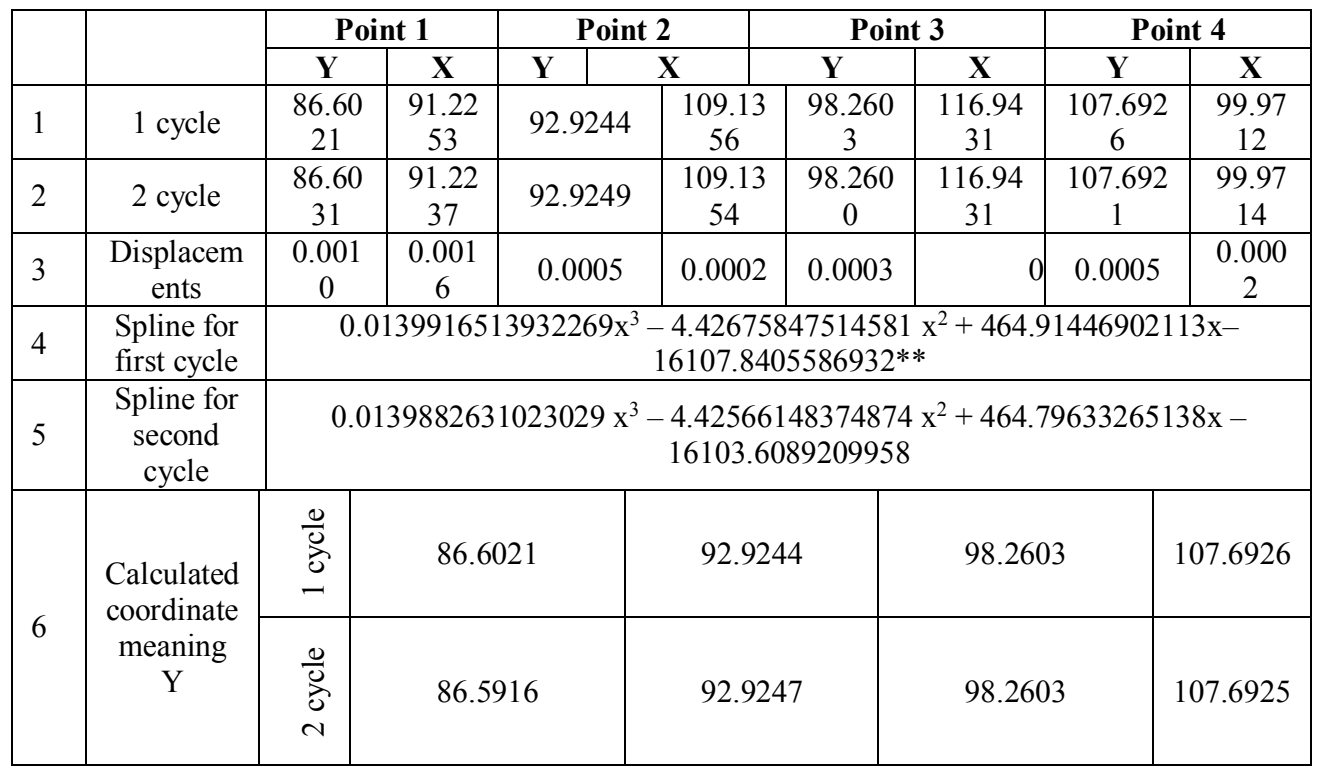


Note: ** - When calculating y coordinates using an approximating spline, keep as many characters as possible; for example, when limiting the number of decimal places to no more than five, the discrepancy is about one and a half meters (85.3358 instead of the actual 86.6021).

Obviously, the calculated Y coordinates in the first cycle coincide with the original ones - rounding errors do not affect. Consider the calculated coordinates for the second cycle and calculate the displacements of the points $\delta c_{i}{ }^{\text {calc }}$ as the difference $y_{i}$ for $k$ and $k+1$ cycle. Consider the difference

$$
\Delta=\delta \mathrm{c}_{\mathrm{i}}^{\text {act }}-\delta \mathrm{c}_{\mathrm{i}}{ }^{\mathrm{calc}},
$$

wherein $\delta \mathrm{c}_{\mathrm{i}}^{\text {act }}$ is displacement determined by measurement results (Table 2).

Table 2. The differences between calculated and actual displacements are more than satisfactory.

\begin{tabular}{|c|c|c|c|c|}
\hline & Point 1 & Point 2 & Point 3 & Point 4 \\
\hline $\begin{array}{c}\text { Actual } \\
\text { displacement } \\
\left(\delta \mathrm{c}_{\mathrm{c}}^{\text {act }}\right)\end{array}$ & 0.0010 & 0.0005 & -0.0003 & -0.0005 \\
\hline $\begin{array}{c}\text { Calculated } \\
\text { displacement } \\
\left(\delta \mathrm{c}_{\mathrm{i}}^{\text {calc }}\right)\end{array}$ & -0.0105 & 0.0003 & 0.0000 & -0.0001 \\
\hline$\Delta$ & 0.0115 & 0.0002 & -0.0003 & -0.0004 \\
\hline
\end{tabular}

Table 2 shows that for the points 2, 3 and 4, the differences between calculated and actual displacements are more than satisfactory. At point 1 the divergence is more than 10 $\mathrm{mm}$ - a value exceeding any permissible error. This is caused by the relative proximity of the so-called "emission" - in the areas of points $\mathrm{x}=56.13$ and $\mathrm{x}=22.97$ function $\mathrm{f}(\mathrm{x})$

$$
f(x)=0.0139916513932269 x^{3}-4.42675847514581 x^{2}+464.91446902113 x-
$$

Reaches a local extremum (at the point $\mathrm{x} \approx 56.13$, the function takes a value close to $\mathrm{f}$ $(\mathrm{x})=-1485)$.

Thus, an intermediate conclusion can be made - the number of significant digits in each of the numbers when performing the calculations should not be less than fifteen, the accuracy of the calculations deteriorates sharply even at a relatively large distance from the extreme points.

In the absence of catastrophic deformations at relatively small distances between the deformation marks (around a few meters), the displacements of the points are quite small. The coefficients at powers of $\mathrm{x}$ in the spline describing the curve are also close to the corresponding spline coefficients for the previous observation cycle. This will simplify the process of calculating the coefficients of the cubic spline.

To find the spline coefficients for the $n+1$ th observation cycle, one can use the coefficients already found for the $n$-th cycle. Indeed let

$$
f_{n}(x)=a_{n} x^{3}+b_{n} x^{2}+c_{n} x+d_{n}, f_{n+1}(x)=a_{n+1} x^{3}+b_{n+1} x^{2}+c_{n+1} x+d_{n+1}
$$

- cubic splines built on the same segment. Due to a small change in the x coordinate, we can write the equality

$$
\begin{gathered}
\mathrm{y}_{\mathrm{n}+1}=\mathrm{f}_{\mathrm{n}+1}(\mathrm{x})=\left(\mathrm{a}_{\mathrm{n}}+\Delta \mathrm{a}\right)(\mathrm{x}+\Delta \mathrm{x})^{3}+\left(\mathrm{b}_{\mathrm{n}}+\Delta \mathrm{b}\right)(\mathrm{x}+\Delta \mathrm{x})^{2}+\left(\mathrm{c}_{\mathrm{n}}+\Delta \mathrm{c}\right)(\mathrm{x}+\Delta \mathrm{x})+\left(\mathrm{d}_{\mathrm{n}}+\Delta \mathrm{d}\right) \\
\mathrm{y}_{\mathrm{n}+1}=\mathrm{a}_{\mathrm{n}} \mathrm{x}^{3}+\mathrm{b}_{\mathrm{n}} \mathrm{x}^{2}+\mathrm{c}_{\mathrm{n}} \mathrm{x}+\mathrm{d}_{\mathrm{n}}+\Delta \mathrm{ax}+\mathrm{a}_{\mathrm{n}} \Delta \mathrm{x}+\Delta \mathrm{bx}+\mathrm{b}_{\mathrm{n}} \Delta \mathrm{x}+\Delta \mathrm{cx}+\mathrm{c}_{\mathrm{n}} \Delta \mathrm{x}+\mathrm{d}_{\mathrm{n}}+\Delta \mathrm{d}+\mathrm{O}\left(\Delta \mathrm{x}^{2,3}\right. \\
\left.\Delta \mathrm{p}^{2,3}\right),
\end{gathered}
$$


wherein $\mathrm{O}\left(\Delta \mathrm{x}^{2,3} ; \Delta \mathrm{p}^{2,3}\right)$ - limited function of the second and third degree increments of the coefficients $\mathrm{p}$ (i.e., $\mathrm{a}, \mathrm{b}$ or $\mathrm{c}$ ) and the argument $\mathrm{x}$. Due to the small increments, it is small value of a higher order than $\Delta \mathrm{x}, \Delta \mathrm{a}, \Delta \mathrm{b}, \Delta \mathrm{c}$ and can be neglected.

Thus,

$$
\mathrm{y}_{\mathrm{n}+1} \approx \mathrm{a}_{\mathrm{n}} \mathrm{x}^{3}+\mathrm{b}_{\mathrm{n}} \mathrm{x}^{2}+\mathrm{c}_{\mathrm{n}} \mathrm{x}+\mathrm{d}_{\mathrm{n}}+\Delta \mathrm{ax}+\mathrm{a}_{\mathrm{n}} \Delta \mathrm{x}+\Delta \mathrm{bx}+\mathrm{b}_{\mathrm{n}} \Delta \mathrm{x}+\Delta \mathrm{cx}+\mathrm{c}_{\mathrm{n}} \Delta \mathrm{x}+\mathrm{d}_{\mathrm{n}}+\Delta \mathrm{d}
$$

The values of $a_{n}, b_{n}, c_{n}, d_{n}, \Delta x$ are known, therefore, we obtain a system of linear equations for $\Delta \mathrm{a}, \Delta \mathrm{b}, \Delta \mathrm{c}$ and $\Delta \mathrm{d}$. As in the case of equation (1), it is necessary to consider groups of four points.

Consider the "flat" case: we study the possibility of approximating surfaces and calculating the coordinates of surface points after its deformation with sub-centimeter accuracy.

As a rule, the location of points on the surface of even a flat facade is irregular, so you should consider splines on an irregular grid. However, in some cases, considering splines on a regular grid is not only acceptable, but also, in some cases, only possible.

At the first step, we consider the linear splines of two variables $\mathrm{S}_{1}=\mathrm{ax}+$ by $+\mathrm{c}$. For an irregular grid defined by points $\left(\mathrm{x}_{\mathrm{i}}, \mathrm{y}_{\mathrm{j}}\right)$, we perform triangulation in the obvious way by building triangles with vertices at these points. In the most general case, when the surface can be defined by a continuous function, $|S(x, y)-f(x, y)|<\omega(f)$, where $\omega(f)$ is the oscillation of the function $\mathrm{z}$ on the triangle $\Omega_{\mathrm{A}}$

$$
\omega(f)=\max \left|f\left(x_{i}, y_{j}\right)-f\left(x_{k}, y_{m}\right)\right|, x_{i}, y_{j}, x_{k}, y_{m} \in \Omega
$$

The order of the error that occurs can be estimated as follows. Suppose for a grid with a distance between the points of not more than $5 \mathrm{~m}$ under the assumption that the deformed surface is (or is well approximated) the surface of a round cylinder of radius $\mathrm{R}=50 \mathrm{~m}$. Consider the position of the measuring device on the extension of the radius passing through the center of the studied surface area. For the domain $\Omega_{\mathrm{A}}:|\mathrm{S}(\mathrm{x}, \mathrm{y})-\mathrm{f}(\mathrm{x}, \mathrm{y})|<62.5$, which corresponds to the position of the approximated point of the surface at the intersection with the radius. Let this radius pass over the edge of a similarly sized region. Then the actual displacement will again not exceed $62.5 \mathrm{~mm}$ (normal to the surface), while the estimate will give the result of a meter order (along the y axis); Thus, firstly, in certain cases the estimate is greatly overestimated, and secondly, it is almost impossible to improve the estimate for some cases: this is an almost exact upper limit of approximation errors on the area.

However, (in the absence of cracks in the physical surface), it can be further assumed that the function defining the surface is piecemeal smooth and has continuous partial derivatives in the entire area of $\Omega_{\mathrm{A}}$. Then the interpolation error can be estimated from the ratios

$$
\begin{gathered}
\left\|S_{1}(x, y)-f(x, y)\right\|_{c} \leq 1 / 3 h^{2}\left\|D^{2} f(x, y)\right\|_{\infty}, \\
\left\|D^{r, s}\left(S_{1}(x, y)-f(x, y)\right)\right\|_{\infty} \leq(2 h / \sin \gamma)\left\|D^{2} f(x, y)\right\|_{\infty}, r+s=1,
\end{gathered}
$$

wherein

$$
\begin{gathered}
\left\|D^{2} f(x, y)\right\|_{\infty}=\operatorname{ess} \sup \left|D^{2} f(x, y)\right|, \\
\left\|S_{1}(x, y)-f(x, y)\right\|_{C}=\max \left|S_{1}(x, y)-f(x, y)\right|,\left(x, y \in \Omega_{A}\right) ;
\end{gathered}
$$

$\mathrm{h}$ - largest side of triangle, $\gamma$ - smallest angle. 


\section{Results}

For the "linear" case, the accuracy of the approximation, as Table 2 suggests, can be increased primarily by a neat choice of the approximating function (it should have no extremums near the approximated points); also, significant contribution is made by error reduction of values rounding in calculations. You can use the approximate expression (2) to calculate a cubic spline at a point.

In the "flat" case, in order to improve the estimation depending on the shape of the triangles selected, one should strive to reduce the larger side and increase the smaller angle (formula (3)). This leads to the need to consider almost equilateral triangles. The value $\left\|\mathrm{D}^{2} \mathrm{f}(\mathrm{x}, \mathrm{y})\right\|_{\infty}$ can be calculated from the recurrence ratios via $\mathrm{B}$-splines; the use of interpolation splines was considered in [5], where the accuracy and convergence rate were also estimated. But in all cases, when using the most general assumptions, the estimate of approximation accuracy by linear local splines is unsatisfactory; the actual deviation of the actual value from the calculated estimate is large enough. The approximation error itself is also quite large even at small (about $5 \mathrm{~m}$ ) distances between points - a consequence of formula (4), therefore, the use of linear splines of two variables in this case seems inappropriate.

\section{Discussion}

Further study of the issue of approximation of point displacement based on the results of observation of several rows of deformation marks requires reference to objects other than linear splines. Part of the problem can be solved by using nonlinear splines ([9] addresses the issue of building surface-approximating splines of order above one). However, any increase in the degree of splines increases the required number of conditions and/or measurements, which completely devalues the decrease in the number of observed points and do not provide the desired decrease in field work.

\section{Conclusions}

Therefore, even for the simplest "linear" case, the problem of calculating the displacements of arbitrary points based on known displacements of points fixed on the surface may have solutions that are substantially dependent on fairly freely selectable initial conditions. These conditions, however, can be strictly regulated if only natural splines are required. In this case, the task has a single solution, but requires knowledge of the values of the derivatives. When using point coordinates determined exclusively in the measurement process, without calculating the values of the spline derivatives, it is possible to obtain a rather accurate approximation, which, however, when approaching the extremum points, is dramatically deteriorated; This requires additional analysis of cubic splines to the extremum. The possibility to improve the accuracy of the displacement calculation lies in the transition to the "spatial" problem - determining the displacement of points when the deformation marks are arranged in several rows along all three coordinates; then displacement of the point $\mathrm{z}=$ $\mathrm{f}(\mathrm{x}, \mathrm{y})$ is a function of two variables. Then, more points at a relatively short distance from the region where the interpolation calculations are made will be used in determining the interpolation functions. Capability to improve accuracy lies in the use of a more complex mathematical apparatus. For example, it seems promising to use the Galerkin-Ritz method or spline minimizers ([10]). 


\section{References}

1. E.A. Nesterenko, Notes of the Mining Institute 204, 127-133 (2013)

2. K.I. Markovich, Bulletin of Siberian state University of geosystems and technologies 24(3), 37-51 (2019)

3. Zhenhua Han, Yingmo Wang, Xinhui Ma, Shugui Liu, Xiaodong Zhang, Guoxiong Zhang, Applied Sciences 7(10) (2017)

4. A.I. Zadorin, N.A. Zadorin, Journal of computational mathematics and mathematical physics 50(2), 221-233 (2010)

5. I.N. Khaimovich, L.S. Klentak, Fundamental research 10-12, 2634-2638 (2013)

6. D.A. Silaev, D.O. Korotaev, Collection of scientific papers of 12th scientific conference. Mathematics. Computer. Education 2, 593-595 (2005)

7. V.A. Fedoruk, SibADI Bulletin 15(2), 306-314 (2018)

8. T.Y. Bugakova, Bulletin of Siberian state University of geosystems and technologies (2019)

9. A.V. Zamyatin, E.A. Zamyatina, Materials of international scientific and practical conference. Rostov state University of civil engineering, Union of builders of Southern Federal district, Association of Don's builders, 348-350 (2015)

10. Qianying Hong, Ming-Jun Lai, Leopold Matamba Messi, Jingyue Wang, J. of Algorithms \& Computational Technology 13 (2019)

11. I. Krasyuk, T. Kirillova, V. Bakharev, B. Lyamin. Proceedings: IOP Conference Series: Materials Science and Engineering. P. 012125. (2019) DOI: 10.1088/1757$899 X / 497 / 1 / 012125$

12. S. Bozhuk, N. Krasnostavskaia, N. Pletneva, T. Maslova. IOP Conference Series: Materials Science and Engineering. 497 (1). P. 012. (2019) DOI: 10.1088/1757$899 \mathrm{X} / 497 / 1 / 012115$

13. D. Gura, M. Kuzyakina, I. Gribkova, IOP Conference Series: Earth and Environmental Science 403(1), 012176 (2019). DOI: 10.1088/1755-1315/403/1/012176 